\title{
Angiogenic and Antiangiogenic Factors in Preterm Neonates Born to Mothers with and without Preeclampsia
}

\author{
Cláudia R. Hentges, MD, $\mathrm{PhD}^{1}$ Rita C. Silveira, MD, $\mathrm{PhD}^{1}$ Renato S. Procianoy, MD, $\mathrm{PhD}^{1}$ \\ ${ }^{1}$ Department of Pediatrics, Newborn Section, Universidade Federal do \\ Rio Grande do Sul and Hospital de Clínicas de Porto Alegre, Porto \\ Alegre-RS, Brazil \\ Address for correspondence Renato S. Procianoy, MD, PhD, \\ Department of Pediatrics, Newborn Section, Universidade Federal do \\ Rio Grande do Sul and Hospital de Clínicas de Porto Alegre, Rua Silva \\ Jardim 1155 \# 701, Porto Alegre, RS 90450-071, Brazil \\ Am J Perinatol 2015;32:1185-1190. \\ (e-mail: rprocianoy@gmail.com).
}

\begin{abstract}
Background Angiogenic and antiangiogenic factors are altered in pregnant women with preeclampsia (PE), but the pattern of expression of these factors in their newborns remains unknown.

Objective This study aims to measure vascular endothelial growth factor (VEGF) and soluble fms-like tyrosine kinase 1 (sFlt-1) levels in preterm neonates born to mothers with PE.

Methods Neonates with birth weight $<2,000 \mathrm{~g}$ and gestational age $\leq 34$ weeks were included and divided into the following two groups: born to mothers with PE and without PE. Blood was collected from neonates within the first 72 hours of life. VEGF and sFlt-1 levels were measured using the enzyme-linked immunosorbent assay method.

Keywords

- angiogenic factors

- preeclampsia

- prematurity

- VEGF

- sFlt-1

- antiangiogenic factors

Results A total of 88 neonates were included ( 37 born to mothers with PE and 51 born to mothers without PE), with a mean gestational age of $29.12 \pm 2.96$ weeks and birth weight of $1,223.80 \pm 417.48 \mathrm{~g}$. In the multivariate analysis, VEGF was $80 \%$ lower and sFlt-1 was 13.48 times higher in the group with PE. sFlt-1 concentration was higher in neonates small for gestational age (SGA) than in those appropriate for gestational age. Conclusion Higher sFlt-1 and lower VEGF levels in the group with PE, as well as higher sFlt-1 levels in SGA neonates, reflect a predominance of antiangiogenic mechanisms in $\mathrm{PE}$ and growth restriction.
\end{abstract}

Preeclampsia (PE) is a multisystem disorder characterized by abnormal vascular response to placentation, which is associated with increased systemic vascular resistance and platelet aggregation and with endothelial cell dysfunction. Proper angiogenesis is essential for the development of the placental vasculature. Its regulation is associated with the balance between angiogenic and antiangiogenic factors, such as vascular endothelial growth factor (VEGF), placental growth factor (PIGF), and soluble VEGF receptor 1 (sVEGFR-1). ${ }^{1,2}$

received

February 9, 2015

accepted after revision

April 6, 2015

published online

May 22, 2015

VEGF is a glycoprotein with potent angiogenic and mitogenic activities and the ability to increase the vascular permeability of endothelial cells. ${ }^{3}$ During pregnancy, VEGF is overproduced by the placenta. ${ }^{4} \mathrm{PIGF}$ is a protein in the VEGF family with similar angiogenic activity, but with weak mitogenic and chemoattractant properties as compared with VEGF. In cells expressing both VEGF and PIGF, formation of VEGF/PIGF heterodimers occurs. The VEGF/PIGF heterodimer is known to be 20 - to 50 -fold less potent than the VEGF homodimer. ${ }^{5}$ In a recent study, we found higher VEGF/PIGF

Copyright $\odot 2015$ by Thieme Medical Publishers, Inc., 333 Seventh Avenue, New York, NY 10001, USA. Tel: +1(212) 584-4662.
DOI http://dx.doi.org/ 10.1055/s-0035-1552932. ISSN 0735-1631. 
heterodimer levels in preterm newborns of preeclamptic mothers. ${ }^{6}$

sVEGFR-1, also known as soluble fms-like tyrosine kinase1 (sFlt-1), is a circulating antiangiogenic protein that binds to VEGF and inhibits its biological activities. Although sFlt- 1 is expressed in endothelial cells and monocytes, it is mainly produced by the placenta during pregnancy. ${ }^{7}$

In the setting of $\mathrm{PE}$, there is poor trophoblast invasion into the maternal spiral arteries with consequent reduction in placental perfusion, leading to placental and fetal hypoxia. In women with established PE, VEGF, and PIGF concentration are reduced and sFlt- 1 concentration is elevated, ${ }^{8-14}$ whereas in normotensive women sFlt- 1 concentration is at a lower level than in preeclamptic women. ${ }^{15-18}$

A few studies have compared levels of angiogenic and antiangiogenic factors (VEGF and sFlt-1) in mothers with and without PE or in umbilical cord blood of their newborns. ${ }^{7,8}$ However, although it is known that VEGF and sFlt-1 levels are altered in preeclamptic pregnant women, no studies have been performed to detect or analyze the pattern of expression of these factors in peripheral blood from preterm neonates born to mothers with PE. Therefore, this study aimed to measure VEGF and sFlt-1 levels in preterm neonates born to mothers with PE.

\section{Material and Methods}

The study was approved by the Research Ethics Committee of Hospital de Clinicas de Porto Alegre, Brazil (protocol no. 110281). Written informed consent was obtained from the neonates' parents or legal guardians before their inclusion in the study.

The study included neonates admitted to the neonatal intensive care unit with birth weight $<2,000 \mathrm{~g}$ and gestational age $\leq 34$ weeks, born to mothers with and without PE. Exclusion criteria were as follows: (1) the neonate was transferred from another institution after 72 hours of life; (2) the neonate died before blood collection; (3) neonates with major congenital anomalies, inborn errors of metabolism, or congenital infections (STORCH screen); (4) neonates born to HIV positive mothers; (5) multiple pregnancies; and (6) mothers with autoimmune disease.

PE was defined as hypertension (blood pressure $\geq 140 /$ $90 \mathrm{~mm} \mathrm{Hg}$ on two separate readings) after 20 weeks' gestation in patients with no previous history of hypertension, accompanied by proteinuria ( $\geq 300 \mathrm{mg} / \mathrm{L}) .{ }^{19}$ Neonates small for gestational age (SGA) were defined as those below the 10th percentile, according to the growth curve developed by Alexander et al. ${ }^{20}$ Sepsis was defined as a positive blood culture, with clinical evidence of infection (changes in breathing pattern, hypo/hyperthermia, and circulatory or gastrointestinal symptoms). Neutropenia was defined as absolute neutrophil count less than $1,000 / \mathrm{mm}^{3}$. Retinopathy of prematurity (ROP) was classified according to Brazilian guidelines for screening and treatment of ROP. ${ }^{21}$ Bronchopulmonary dysplasia (BPD) was considered when the newborn was oxygen dependent for more than 28 days, and had a chest X-ray compatible with BPD.
Blood samples were collected from all neonates within the first 72 hours of life. No samples were collected exclusively for the study; an additional $1 \mathrm{~mL}$ of blood was collected during routine blood draws. All neonates were followed up on their development from inclusion in the study until hospital discharge or death.

Blood samples were drawn into tubes containing ethylenediaminetetraacetic acid and centrifuged at 5,000 rpm for 10 minutes at $4^{\circ} \mathrm{C}$. Plasma was separated and stored at $-80^{\circ} \mathrm{C}$ in Eppendorf tubes. VEGF and sFlt-1 measurements were performed using the enzyme-linked immunosorbent assay (ELISA) according to the manufacturer's instructions (R\&D Systems Inc, Minneapolis, MN), as performed in previous studies. ${ }^{7,8,22}$ The limit of detection for VEGF was $5 \mathrm{pg} / \mathrm{mL}$, with an intra- and interassay coefficient of variation of 6.7 and $8.8 \%$, respectively. The limit of detection for sFlt- 1 was $5 \mathrm{pg} /$ $\mathrm{mL}$ with an intra- and interassay coefficient of variation of 3.8 and $7.0 \%$, respectively. All measurements were performed in duplicate. All samples were processed and analyzed in the laboratory of molecular biology of the institution.

Sample size calculation was based on the study by Catarino et al. ${ }^{7}$ Assuming a level of significance of 5 and $95 \%$ power, a minimum sample of 60 preterm neonates was required, with 30 patients in each group (with and without PE).

Statistical analysis was performed using the Statistical Package for the Social Sciences (SPSS) version 16.0 (SPSS Inc., Chicago, IL). The level of significance was set at $p<0.05$. Data are expressed as median and interquartile range or mean and standard deviation. Statistical differences between groups were determined by the chi-square test, $t$ test, or Mann-Whitney test. Factors found to be significant $(p<0.05)$ in the univariate analysis were used as control variables in the multivariate analysis to compare groups. Serial VEGF and sFlt-1 measurements were compared using a generalized estimating equation with gamma distribution and a log link function (after multiple comparisons with Bonferroni correction). Graphs were presented as a bee swarm plot using $\mathrm{R}$ package version $0.1 .1 .^{23}$

\section{Results}

A total of 88 neonates were included in the study (37 born to mothers with and 51 born to mothers without PE), with a mean gestational age of $29.12 \pm 2.96$ weeks and mean birth weight of $1,223.80 \pm 417.48 \mathrm{~g}$. The group with PE showed lower gestational age, shorter duration of premature rupture of membranes (PROM), and higher incidence of cesarean delivery and of SGA than the control group ( $\mathbf{- T a b l e ~} \mathbf{1}$ ).

Plasma VEGF levels were lower in neonates born to mothers with PE (32.45 pg/mL [6.36-85.75]) than in controls (82.38 pg/mL [35-130.03]) $(p=0.001)$ ( - Fig. 1), while sFlt-1 levels were higher in neonates born to mothers with PE (1338.57 pg/mL [418.8-3472.24]) than in controls (318.13 $\mathrm{pg} / \mathrm{mL}$ [182.03-453.66]) $(p<0.001)$ ( - Fig. 2). These findings remained significant in the multivariate analysis, with VEGF levels $80 \%$ lower and sFlt-1 levels 13.48 times higher in neonates born to mothers with PE (-Tables 2 and 3). VEGF and sFlt-1 were analyzed separately in the multivariate 
Table 1 Characteristics of preterm neonates according to the presence of maternal preeclampsia

\begin{tabular}{|c|c|c|c|}
\hline & Preeclampsia $(n=37)$ & Control $(n=51)$ & $p$ \\
\hline $\mathrm{GA}(\mathrm{wks})^{1}$ & $30.81 \pm 2.34$ & $29.43 \pm 2.80$ & 0.014 \\
\hline PROM $(h)^{2}$ & $0(0-0)$ & $0(0-9.5)$ & 0.001 \\
\hline Cesarean delivery $[n(\%)]^{3}$ & 35 (94.6) & $23(45.1)$ & $<0.001$ \\
\hline Birth weight $(\mathrm{g})$ & $1,215.54 \pm 423.08$ & $1,229.78 \pm 417.48$ & 0.876 \\
\hline Male & $18(48.6 \%)$ & $24(47.1 \%)$ & 1 \\
\hline APGAR $<7$ at 5 min & $10(27 \%)$ & $7(13.7 \%)$ & 0.198 \\
\hline SGA & $26(70.3 \%)$ & $11(21.6 \%)$ & $<0.001$ \\
\hline SNAPPE II & $18(2.5-47)$ & $14(0-27)$ & 0.231 \\
\hline Use of CPAP & 19 (51.4\%) & $21(41.2 \%)$ & 0.466 \\
\hline Use of MV & 17 (45.9\%) & $26(51 \%)$ & 0.802 \\
\hline RDS & $18(48.6 \%)$ & $23(45.1 \%)$ & 0.910 \\
\hline BPD & $6(20 \%)$ & $17(38.6 \%)$ & 0.125 \\
\hline Caffeine use & $7(18.9 \%)$ & $8(15.7 \%)$ & 0.912 \\
\hline Sepsis & $1(2.7 \%)$ & 0 & 0.420 \\
\hline Use of vasopressors & $2(5.4 \%)$ & $7(13.7 \%)$ & 0.293 \\
\hline Red blood cell transfusion & $2(5.4 \%)$ & $3(5.9 \%)$ & 1 \\
\hline Platelets $<150.000$ & $8(21.6 \%)$ & $7(13.7 \%)$ & 0.493 \\
\hline Neutropenia & $5(13.5 \%)$ & $5(9.8 \%)$ & 0.736 \\
\hline PDA & $3(8.1 \%)$ & $6(11.8 \%)$ & 0.728 \\
\hline Seizures & $1(2.7 \%)$ & $1(2 \%)$ & 1 \\
\hline IVH grades 3 and 4 & $1(2.7 \%)$ & $5(9.8 \%)$ & 0.39 \\
\hline Treatable ROP & 0 & $1(2 \%)$ & 1 \\
\hline Death & $7(18.9 \%)$ & $11(21.6 \%)$ & 0.971 \\
\hline
\end{tabular}

Abbreviations: BPD, bronchopulmonary dysplasia; CPAP, continuous positive airway pressure; GA, gestational age; IVH, intraventricular hemorrhage; MV, mechanical ventilation; PDA, patent ductus arteriosus; PROM, premature rupture of membranes; RDS, respiratory distress syndrome; ROP, retinopathy of prematurity; SGA, small for gestational age.

${ }^{1} t$-test for independent samples.

${ }^{2}$ Mann-Whitney test.

${ }^{3}$ Chi-square test.

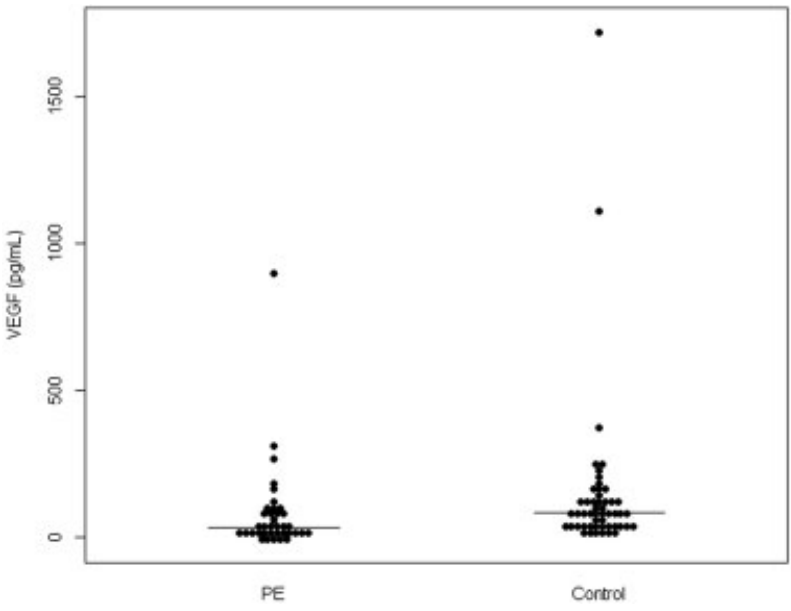

Fig. 1 Plasma VEGF levels in the groups with preeclampsia (PE) and without PE (control). The horizontal bar represents the median value ( $p=0.001$, Mann-Whitney test). VEGF, vascular endothelial growth factor.

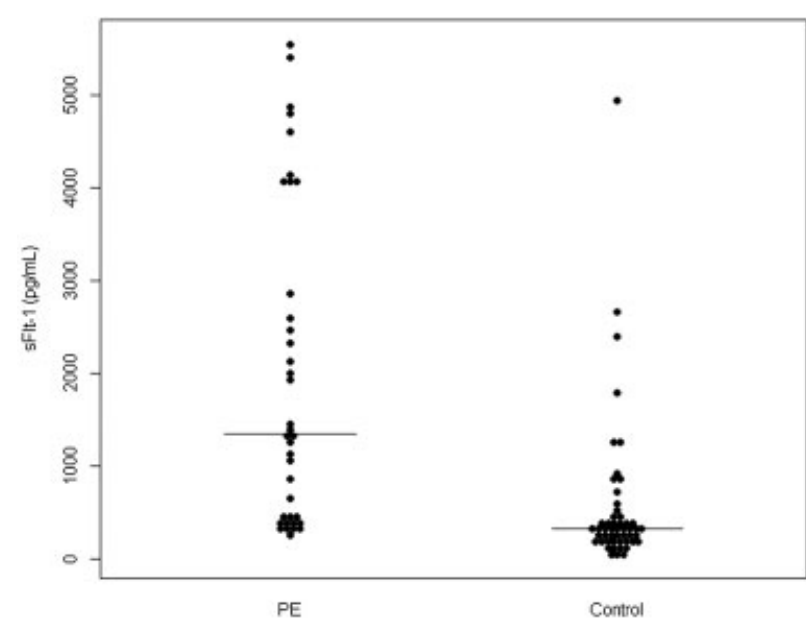

Fig. 2 Plasma sFlt-1 levels in the groups with preeclampsia (PE) and without $\mathrm{PE}$ (control). The horizontal bar represents the median value $(p<0.001$, Mann-Whitney test).sFlt-1, soluble fms-like tyrosine kinase-1. 
Table 2 Multivariate analysis of risk factors associated with neonates born to preeclamptic mothers, including VEGF

\begin{tabular}{|l|l|l|l|}
\hline & OR & $95 \% \mathrm{Cl}$ & $\boldsymbol{p}$ \\
\hline GA $^{1}$ & 1.10 & $0.85-1.44$ & 0.47 \\
\hline PROM & 0.95 & $0.78-1.00$ & 0.48 \\
\hline Vaginal delivery & 0.04 & $0.005-0.19$ & $<0.001$ \\
\hline SGA & 6.06 & $1.69-25.12$ & 0.008 \\
\hline VEGF & 0.20 & $0.04-0.76$ & 0.02 \\
\hline
\end{tabular}

Abbreviations: 95\% Cl, 95\% confidence interval; GA, gestational age; OR, odds ratio; PROM, premature rupture of membranes; SGA, small for gestational age; VEGF, vascular endothelial growth factor.

${ }^{1}$ Variables with significant differences in the univariate analysis.

model, as these two factors are closely related and could not be included in the same model.

Comparing VEGF and SFlt-1 levels regarding appropriate for gestational age (AGA) and SGA status in all population, sFlt-1 levels were higher in SGA neonates $(1,044.94 \mathrm{pg} / \mathrm{mL}$ [290.64-3,472.24]) than in neonates AGA $(372.67 \mathrm{pg} / \mathrm{mL}$ [236.75-860.14]) $(p=0.013)$. As for VEGF, SGA neonates had plasma levels similar to those of AGA neonates (45.64 [6.36-105.67] vs. 70.45 [29.17-126.60] pg/mL) $(p=0.183)$.

\section{Discussion}

In our study, plasma sFlt-1 levels were four times higher in preterm neonates exposed to maternal PE than in controls, whereas plasma VEGF levels were 2.5 times lower. Even after multivariate analysis, higher sFlt- 1 and lower VEGF plasma concentrations were maintained in preterm exposed to maternal PE. Previous studies measuring these substances in umbilical cord blood from neonates exposed to PE showed similar results. ${ }^{7,8}$

Increased sFlt-1 concentration is known to be associated with decreased biologically active VEGF concentration, as sFlt-1 binds to VEGF, leading to extremely low levels of free VEGF. $^{2}$ In vitro conditions, VEGF induces cytotrophoblast invasion, an effect that is blocked by addition of sFlt- $1 .{ }^{4}$

Table 3 Multivariate analysis of risk factors associated with neonates born to preeclamptic mothers, including sFlt-1

\begin{tabular}{|l|l|l|l|}
\hline & OR & $95 \% \mathrm{Cl}$ & $p$ \\
\hline GA $^{1}$ & 1.20 & $0.90-1.66$ & 0.22 \\
\hline PROM & 0.96 & $0.81-1$ & 0.50 \\
\hline Vaginal delivery & 0.13 & $0.018-0.68$ & 0.026 \\
\hline SGA & 4.07 & $1.08-17.05$ & 0.042 \\
\hline sFlt-1 & 13.48 & $2.66-98.96$ & 0.004 \\
\hline
\end{tabular}

Abbreviations: $95 \% \mathrm{Cl}$, 95\% confidence interval; GA, gestational age; OR, odds ratio; PROM, premature rupture of membranes; SGA, small for gestational age; sFlt-1, soluble fms-like tyrosine kinase-1.

${ }^{1}$ Variables with significant differences in the univariate analysis.
VEGF is a potent stimulator of endothelial cell proliferation, promoting angiogenesis. Its receptor, sFlt-1, binds VEGF with high affinity and inhibits its mitogenic activity. ${ }^{24}$ Hypoxia increases sFlt-1 expression in trophoblasts, leading to decreased levels of free VEGF and triggering an antiangiogenic state. ${ }^{1}$ In the setting of $\mathrm{PE}$, placental cells are exposed to reduced perfusion because of inadequate trophoblast invasion, leading to elevated sFlt-1 concentration.

The placenta is the main source of sFlt- 1 , and studies suggest that transplacental transfer of this substance to the fetus is more important than fetal production of sFlt- $1 .{ }^{7,8}$ As for VEGF, it is expressed at sites of active angiogenesis, as it occurs in the fetus. When comparing maternal and fetal (via umbilical cord blood) concentrations, higher fetal concentrations have been observed. ${ }^{7}$ We suggest that determination of those factor in umbilical blood may not reflect the actual situation in peripheral neonatal blood sample as it was described for other factors, ${ }^{25,26}$ once that there is neonatal participation in the production of both factors. A strong point of our study is the fact that to the best of our knowledge, no study published to date has compared VEGF and sFlt-1 levels in blood from preterm neonates born to mothers with PE.

Regarding the clinical findings, the higher incidence of cesarean delivery and, consequently, shorter duration of PROM may be attributed to an indication for pregnancy interruption in severe cases, ${ }^{27,28}$ although we did not classify PE in our study patients. Surprisingly, there was no difference between groups in the incidence of neutropenia, thrombocytopenia, respiratory distress syndrome, $\mathrm{BPD}$, and treatable ROP, in disagreement with previous reports. ${ }^{29-36}$ We did not follow our patients up to discharge to determine the duration of the antiangiogenic status would last. However, our study was designed only to evaluate factors involved in angiogenesis just after birth, which is a limitation of the study.

Intrauterine growth restriction (IUGR) is characterized by an altered placental angiogenesis, leading to a reduced supply of oxygen, and nutrients to the fetus. It is described in newborns that there is an association between low sFlt- 1 levels in the beginning of pregnancy followed by a significant increase along the gestation and intrauterine growth retardation. ${ }^{37}$ It is possible that exposure of SGA neonates to elevated sFlt-1 concentrations leads to vascular effects that, in the long term, may play an important role in the association of low birth weight with risk of cardiovascular disease in the future. ${ }^{37,38}$ We found no difference in VEGF levels when SGA and AGA neonates were compared. This finding confirms previous study that showed an inversely association of umbilical cord blood VEGF levels and birth weight percentile and birth weight, but not when VEGF levels are determined in peripheral blood in term newborns. ${ }^{39}$ However, sFlt- 1 levels were 2.8 times higher in our SGA neonates, compared with AGA neonates, which is consistent with findings previously reported in full-term neonates. ${ }^{24}$ In the setting of PE, the placenta produces high levels of sFlt- $1 .{ }^{4}$ However, elevated sFlt- 1 concentration can also be observed in cases of chronic intrauterine hypoxia without PE. ${ }^{24}$ 


\section{Conclusion}

Higher sFlt-1 and lower VEGF levels were found in preterm neonates exposed to PE, as well as higher sFlt-1 levels in SGA delivery, reflecting a predominance of antiangiogenic mechanisms in PE and IUGR.

\section{Conflicts of Interest}

The authors declare no conflicts of interest.

\section{Funding Source}

We received funding for Research and Event Promotion (FIPE-HCPA) and Research Support Foundation of Rio Grande do Sul (FAPERGS) (IRB No. 11-0281).

\section{Acknowledgments}

We thank Vania Naomi Hirakata and Luciano Santos Pinto Guimarães for their assistance in data analysis, and Prof. Dr. Úrsula Matte for her laboratory assistance.

\section{References}

1 Silasi M, Cohen B, Karumanchi SA, Rana S. Abnormal placentation, angiogenic factors, and the pathogenesis of preeclampsia. Obstet Gynecol Clin North Am 2010;37(2):239-253

2 Lam C, Lim KH, Karumanchi SA. Circulating angiogenic factors in the pathogenesis and prediction of preeclampsia. Hypertension 2005;46(5):1077-1085

3 Muy-Rivera M, Vadachkoria S, Woelk GB, Qiu C, Mahomed K, Williams MA. Maternal plasma VEGF, sVEGF-R1, and PIGF concentrations in preeclamptic and normotensive pregnant Zimbabwean women. Physiol Res 2005;54(6):611-622

4 Cerdeira AS, Karumanchi SA. Angiogenic factors in preeclampsia and related disorders. Cold Spring Harb Perspect Med 2012;2(11): pii: a006585

5 Ribatti D. The discovery of the placental growth factor and its role in angiogenesis: a historical review. Angiogenesis 2008;11(3): 215-221

6 Hentges CR, Silveira RC, Ferrelli RS, Procianoy RS. Influence of maternal pre-eclampsia on VEGF/PIGF heterodimer levels in preterm infants. J Matern Fetal Neonatal Med 2014;14:1-6

7 Catarino C, Rebelo I, Belo L, et al. Fetal and maternal angiogenic/ anti-angiogenic factors in normal and preeclamptic pregnancy. Growth Factors 2009;27(6):345-351

8 Staff AC, Braekke K, Harsem NK, Lyberg T, Holthe MR. Circulating concentrations of sFlt1 (soluble fms-like tyrosine kinase 1) in fetal and maternal serum during preeclampsia. Eur J Obstet Gynecol Reprod Biol 2005;122(1):33-39

9 Rana S, Karumanchi SA, Levine RJ, et al. Sequential changes in antiangiogenic factors in early pregnancy and risk of developing preeclampsia. Hypertension 2007;50(1):137-142

10 Erez O, Romero R, Espinoza J, et al. The change in concentrations of angiogenic and antiangiogenic factors in maternal plasma between the first and second trimesters in risk assessment for the subsequent development of preeclampsia and small-forgestational age. J Matern Fetal Neonatal Med 2008;21(5): 279-287

11 Maynard SE, Min JY, Merchan J, et al. Excess placental soluble fmslike tyrosine kinase 1 (sFlt1) may contribute to endothelial dysfunction, hypertension, and proteinuria in preeclampsia. J Clin Invest 2003;111(5):649-658
12 Levine RJ, Maynard SE, Qian C, et al. Circulating angiogenic factors and the risk of preeclampsia. N Engl J Med 2004;350(7):672-683

13 Vatten LJ, Eskild A, Nilsen TI, Jeansson S, Jenum PA, Staff AC. Changes in circulating level of angiogenic factors from the first to second trimester as predictors of preeclampsia. Am J Obstet Gynecol 2007;196(3):239.e1-239.e6

14 Troisi R, Braekke K, Harsem NK, Hyer M, Hoover RN, Staff AC. Blood pressure augmentation and maternal circulating concentrations of angiogenic factors at delivery in preeclamptic and uncomplicated pregnancies. Am J Obstet Gynecol 2008;199(6):653.e1-653.e10

15 Levine RJ, Lam C, Qian C, et al; CPEP Study Group. Soluble endoglin and other circulating antiangiogenic factors in preeclampsia. N Engl J Med 2006;355(10):992-1005

16 Staff AC, Harsem NK, Braekke K, Hyer M, Hoover RN, Troisi R. Maternal, gestational and neonatal characteristics and maternal angiogenic factors in normotensive pregnancies. Eur J Obstet Gynecol Reprod Biol 2009;143(1):29-33

17 Sunderji S, Gaziano E, Wothe D, et al. Automated assays for sVEGF $\mathrm{R} 1$ and PIGF as an aid in the diagnosis of preterm preeclampsia: a prospective clinical study. Am J Obstet Gynecol 2010;202(1):40. e1-40.e7

18 Furuya M, Kurasawa K, Nagahama K, et al. Disrupted balance of angiogenic and antiangiogenic signalings in preeclampsia. J Pregnancy 2011;2011:123717

19 Sibai B, Dekker G, Kupferminc M. Pre-eclampsia. Lancet 2005; 365(9461):785-799

20 Alexander GR, Himes JH, Kaufman RB, Mor J, Kogan M. A United States national reference for fetal growth. Obstet Gynecol 1996; 87(2):163-168

21 Zin A, Florêncio T, Fortes Filho JB, et al; Brazilian Society of Pediatrics, Brazilian Council of Ophthalmology and Brazilian Society of Pediatric Ophthalmology. Brazilian guidelines proposal for screening and treatment of retinopathy of prematurity (ROP). Arq Bras Oftalmol 2007;70(5):875-883

22 Pieh C, Agostini H, Buschbeck C, et al. VEGF-A, VEGFR-1, VEGFR2 and Tie2 levels in plasma of premature infants: relationship to retinopathy of prematurity. $\mathrm{Br} \mathrm{J}$ Ophthalmol 2008;92(5): 689-693

23 Eklund A. Beeswarm: The bee swarm plot, an alternative to stripchart. R package version 0.1.1, 2011. Available at: http:// CRAN.R-project.org/package=beeswarm. Accessed February 2, 2015

24 Boutsikou T, Malamitsi-Puchner A, Economou E, Boutsikou M, Puchner KP, Hassiakos D. Soluble vascular endothelial growth factor receptor-1 in intrauterine growth restricted fetuses and neonates. Early Hum Dev 2006;82(4):235-239

25 van Beem RT, Verloop RE, Kleijer M, et al. Blood outgrowth endothelial cells from cord blood and peripheral blood: angiogenesis-related characteristics in vitro. J Thromb Haemost 2009;7(1): 217-226

26 Khan I, Zhang L, Mohammed M, et al. Effects of Wharton's jellyderived mesenchymal stem cells on neonatal neutrophils. J Inflamm Res 2015;8:1-8

27 Mashiloane CD, Moodley J. Induction or caesarean section for preterm pre-eclampsia? J Obstet Gynaecol 2002;22(4):353-356

28 Zhang Y, Li W, Xiao J, Chen S. The complication and mode of delivery in Chinese women with severe preeclampsia: a retrospective study. Hypertens Pregnancy 2014;33(3):283-290

29 Jelin AC, Cheng YW, Shaffer BL, Kaimal AJ, Little SE, Caughey AB. Early-onset preeclampsia and neonatal outcomes. J Matern Fetal Neonatal Med 2010;23(5):389-392

30 Wang A, Holston AM, Yu KF, et al. Circulating anti-angiogenic factors during hypertensive pregnancy and increased risk of respiratory distress syndrome in preterm neonates. J Matern Fetal Neonatal Med 2012;25(8):1447-1452

31 Ozkan H, Cetinkaya M, Koksal N. Increased incidence of bronchopulmonary dysplasia in preterm infants exposed to preeclampsia. J Matern Fetal Neonatal Med 2012;25(12):2681-2685 
32 Tang JR, Karumanchi SA, Seedorf G, Markham N, Abman SH. Excess soluble vascular endothelial growth factor receptor-1 in amniotic fluid impairs lung growth in rats: linking preeclampsia with bronchopulmonary dysplasia. Am J Physiol Lung Cell Mol Physiol 2012;302(1):L36-L46

33 Hansen AR, Barnés CM, Folkman J, McElrath TF. Maternal preeclampsia predicts the development of bronchopulmonary dysplasia. J Pediatr 2010;156(4):532-536

34 Ozkan H, Cetinkaya M, Koksal N, Ozmen A, Yıldız M. Maternal preeclampsia is associated with an increased risk of retinopathy of prematurity. J Perinat Med 2011;39(5):523-527

35 Tsao PN, Wei SC, Su YN, Chou HC, Chen CY, Hsieh WS. Excess soluble fms-like tyrosine kinase 1 and low platelet counts in premature neonates of preeclamptic mothers. Pediatrics 2005; 116(2):468-472
36 Procianoy RS, Silveira RC, Mussi-Pinhata MM, et al; Brazilian Network on Neonatal Research. Sepsis and neutropenia in very low birth weight infants delivered of mothers with preeclampsia. J Pediatr 2010;157(3):434-438, 438.e1

37 Åsvold BO, Vatten LJ, Romundstad PR, Jenum PA, Karumanchi SA, Eskild A. Angiogenic factors in maternal circulation and the risk of severe fetal growth restriction. Am J Epidemiol 2011;173(6):630-639

38 Taki A, Abe M, Komaki M, et al. Expression of angiogenesis-related factors and inflammatory cytokines in placenta and umbilical vessels in pregnancies with preeclampsia and chorioamnionitis/ funisitis. Congenit Anom (Kyoto) 2012;52(2):97-103

39 Malamitsi-Puchner A, Boutsikou T, Economou E, et al. Vascular endothelial growth factor and placenta growth factor in intrauterine growth-restricted fetuses and neonates. Mediators Inflamm 2005;2005(5):293-297 